\title{
A mechanism for morphogen-controlled domain growth
}

\author{
R. E. Baker • P. K. Maini
}

Received: 14 March 2006 / Revised: 31 October 2006 /

Published online: 16 December 2006

(C) Springer-Verlag 2006

\begin{abstract}
Many developmental systems are organised via the action of graded distributions of morphogens. In the Drosophila wing disc, for example, recent experimental evidence has shown that graded expression of the morphogen Dpp controls cell proliferation and hence disc growth. Our goal is to explore a simple model for regulation of wing growth via the Dpp gradient: we use a system of reaction-diffusion equations to model the dynamics of Dpp and its receptor $\mathrm{Tkv}$, with advection arising as a result of the flow generated by cell proliferation. We analyse the model both numerically and analytically, showing that uniform domain growth across the disc produces an exponentially growing wing disc.
\end{abstract}

Keywords Drosophila $\cdot$ Domain growth · Morphogen gradient .

Mathematical model

\section{Introduction}

The development of any organism begins with a handful of cell types arranged in a crude manner. As development proceeds these cells divide, differentiate and migrate, eventually producing a highly-organised system comprising many

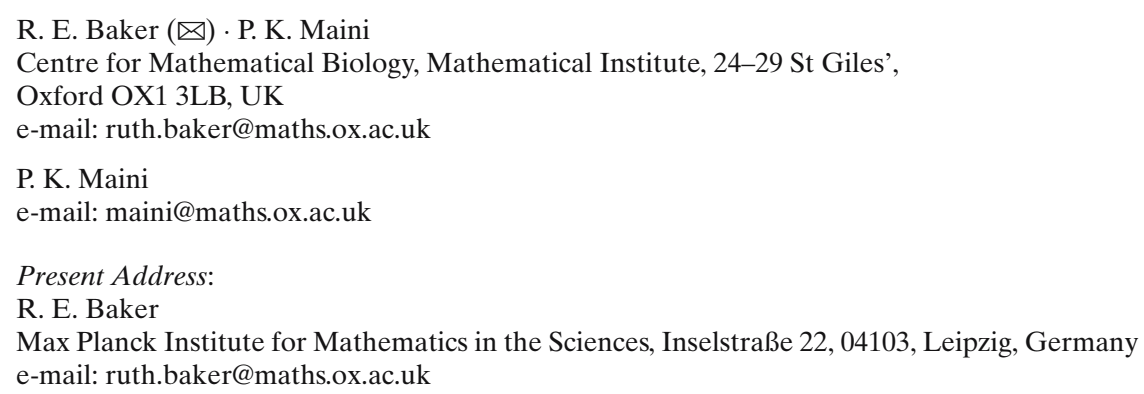


different cell types, each with specialised function. Much experimental and theoretical research has been dedicated to elucidating the mechanisms underlying development: such mechanisms must be sufficiently robust to cope with genetic variation and environmental noise; yet able to create the kind of fine-detailed patterning observed in the fully-developed embryo.

The role of morphogens in development has been long since documented: a widely accepted definition of a morphogen is that of a diffusible substance which provides spatial and temporal information during development via a concentration gradient. Wolpert [28] first proposed this mechanism for patterning in 1969 using his positional information model: he supposes a morphogen produced at a localised source diffuses across a target field to set up an extracellular gradient. Cells determine their position within the field by interpretation of the morphogen gradient, activating specific programs of differentiation at discrete morphogen thresholds. In this way we see that pattern formation using positional information relies on two mechanisms: (i) specification of the positional information via a morphogen gradient; (ii) interpretation of the positional information by cells in the morphogenetic field [29].

Morphogen gradients play important roles in numerous developmental mechanisms: neuronal cell fate in the developing central nervous system (CNS) is controlled by gradients of Sonic Hedgehog (Shh) [3]; formation of the dorsal-ventral (DV) axis in Drosophila is controlled by gradients of Screw (Scw) and Decapentaplegic (Dpp) [8,17,27]; antero-posterior (AP) patterning in the chick wing is controlled by a gradient of Shh arising from a polarising region at the posterior edge of the limb bud [24]; to name but a few.

Much of our knowledge about morphogen gradients has come from experimental observations on the invertebrate Drosophila and several morphogen gradients have been identified as crucial to Drosophila development. Alongside the aforementioned role of Dpp: Bicoid is known to be the primary determinant of anterior body pattern [14]; Hunchback has been shown to play a role in inducing striped gene expression in the posterior half of the embryo [14]; and Dpp has been shown to act in a concentration-dependent manner to activate various target genes in the Drosophila wing disc [26].

Correspondingly there has been much theoretical investigation of the formation and robustness of morphogen gradients in Drosophila (see, for theoretical models, $[2,7,10,11,13])$. In particular, Lander and co-workers $[12,13]$ discuss the mechanisms via which a stable gradient of Dpp may be maintained in the wing disc, exploring the possible roles of receptor binding, internalisation and endocytosis, and ligand diffusion in creating long range gradients.

More recently, it has been demonstrated that a gradient of Dpp is responsible for controlling growth in the Drosophila wing disc [19,18,21] and it is this phenomenon in which we will be interested in this paper. In [21], Rogulja and co-workers report on the use of two mechanisms for controlling domain growth: the first relies on the juxtaposition of cells with different levels of Dpp activity, so that cell proliferation only occurs in a region where the Dpp gradient is suitably steep; the second relies on reduced Dpp activity (away from the morphogen source) and the corresponding upregulation of brinker [18]. 


\subsection{Aims and outline}

Although there have been many models describing Dpp gradient formation and maintenance, we are not familiar with any which address the role of this morphogen in driving cell proliferation and hence domain growth. The aim of this paper is to establish an initial model to describe Dpp activity in the Drosophila wing disc and the resulting growth of the disc. The mathematical framework developed in this paper will, in the future, allow us to investigate key unanswered questions surrounding this mechanism of domain growth and to make experimental predictions which could be used to further understanding in the area.

To model the phenomena outlined above we will use a system of reactiondiffusion equations, with advection arising as a result of the flow generated by cell proliferation in the wing. In Sect. 2 we outline the experimental evidence: detailing the biochemical pathways which are involved in Dpp gradient formation and maintenance, and the mechanisms via which this gradient leads to cell proliferation. In Sect. 3 we outline the main mathematical techniques that will be used to model the Dpp gradient in Sect. 4. We solve the model numerically and in Sect. 5 we make some simplifying approximations which allow us to apply analytical techniques to the model. We conclude in Sect. 6 with a discussion of the model and outline future work and ideas.

\section{The role of Dpp in Drosophila wing growth}

The Drosophila wing begins life as a disc of about 40 cells which grows, over the span of about four days, into a monolayered epithelial sac consisting of around 50,000 cells [21]. A number of genes are known to play roles in patterning and shaping the disc and one such gene is Dpp [6,18,19,22].

Dpp is produced along the AP compartment boundary (see Fig. 1) and it spreads out from its site of synthesis, thereby forming a gradient. As mentioned in Sect. 1, this Dpp gradient has recently been shown to control cell proliferation in the wing: in medial regions of the wing, close to the AP compartment boundary, the gradient of Dpp activity is steep and Rogulja and co-workers report that this juxtaposition of cells experiencing different levels of Dpp activity is necessary and sufficient for cell proliferation [21]. In more lateral regions of the wing, low levels of Dpp are unable to repress brinker activity and this also leads to cell proliferation [18,21]. It has been shown that: growth occurs uniformly across the wing; Dpp mutants show severly impaired growth; overexpression of Dpp promotes wing overgrowth; and clonal activation can change the growth regions within the disc [21].

The Dpp receptor, Thickveins (Tkv), has been shown to play a role in shaping the Dpp gradient: Dpp binds reversibly to Tkv and once bound it is unable to diffuse but can be internalised and degraded, or subject to endocytotic trafficking $[9,23]$. Dpp, on the other hand, has been shown to negatively regulate Tkv receptor expression [15]. Tkv also plays a major role in transducing the Dpp 


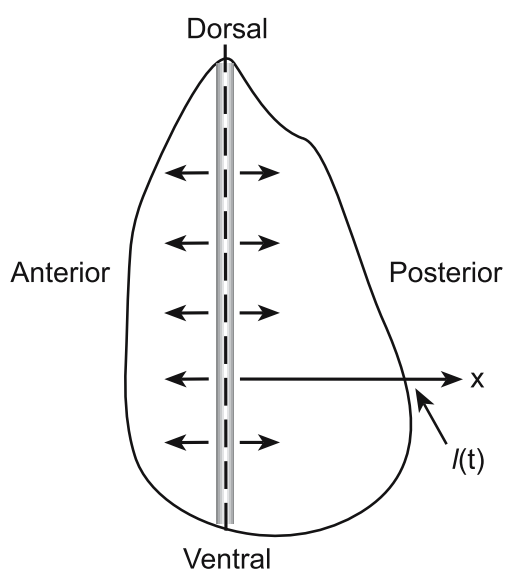

Fig. 1 An illustration of the Drosophila wing disc. The AP compartment boundary is indicated by the dashed line and the source of Dpp by the shaded strip following the compartment boundary. Dpp diffuses from its site of synthesis into both compartments, in a medial to lateral direction. The anterior compartment lies to the left-hand side of the boundary and the posterior compartment to the right-hand side. $x$ marks the growth axis considered in Sect. 3.3 and $l(t)$ marks the position of the disc boundary

signal by phosphorylating the major signal transducer Mothers against Dpp (Mad), which then translocates into the nucleus [9,22,25].

Opposing views exist as to the importance of diffusion versus endocytotic trafficking and the rate of gradient formation (possibly limited due to space constrictions and the level of Tkv receptors). The implications of these factors with respect to modelling this phenomena will be discussed in Sect. 6 .

\section{Modelling reaction-diffusion systems on growing domains}

Here we outline some of the main mathematical techniques that will be used to model the Dpp gradient in this work. We will use a system of partial differential equations (PDEs) to describe the dynamics of the ligand Dpp, and the free and bound forms of its receptor, Tkv. We will outline derivation of the flow generated in the wing disc due to growth and suggest a suitable frame of reference for analysing our models.

\subsection{Derivation of evolution equations}

Following Crampin and co-workers [4], we consider the evolution of $n$ chemical species $c_{1}, \ldots, c_{n}$ reacting and diffusing on a time-varying domain $\underline{\Omega}(t)$. For any elemental volume $\underline{V}(t)$ in the domain $\underline{\Omega}(t)$ we can write down the following system of conservation equations:

$$
\frac{\mathrm{d}}{\mathrm{d} t} \int_{\underline{V}(t)} \underline{c}(\underline{x}, t) \mathrm{d} \underline{x}=\int_{\underline{V}(t)}[-\nabla \cdot \underline{j}+\underline{R}(\underline{c})] \mathrm{d} \underline{x},
$$


where $\underline{c}$ is a column vector of length $n$ representing chemicals $c_{1}, \ldots, c_{n} . j$ is the flux and $\underline{R}$ the reaction term, which includes production and decay as well as interactions between the individual chemicals. Via the Reynolds Transport Theorem [1], the left-hand side of Eq. (1) can be evaluated to give

$$
\frac{\mathrm{d}}{\mathrm{d} t} \int_{\underline{\underline{V}(t)}} \underline{c}(\underline{x}, t) \mathrm{d} \underline{x}=\int_{\underline{V}(t)}\left[\frac{\partial \underline{c}}{\partial t}+\nabla \cdot(\underline{a} \underline{c})\right] \mathrm{d} \underline{x},
$$

where $\underline{a}(\underline{x}, t)$ is the flow due to domain growth.

These results hold for any elemental volume $\underline{V}(t)$ moving in the flow and so we argue that they must hold everywhere in the volume $\underline{\Omega}(t)$. Assuming Fickian flux, $j=-\mathcal{D} \nabla \underline{c}$ where $\mathcal{D}$ is a diagonal matrix representing the diffusivities of individual chemicals, we arrive at the following system of evolution equations:

$$
\frac{\partial \underline{c}}{\partial t}+\nabla \cdot(\underline{a} \underline{c})=\mathcal{D} \nabla^{2} \underline{c}+\underline{R}(\underline{c}), \quad \underline{x} \in \underline{\Omega}(t), \quad t \in[0, \infty) .
$$

The system is closed by specifying initial and boundary conditions of the form

$$
\underline{c}(\underline{x}, 0)=\underline{c}_{0}(\underline{x}) \quad \text { and } \quad \underline{\Phi}(\underline{x}, t) \frac{\partial \underline{c}}{\partial n}+\underline{\Psi}(\underline{x}, t) \underline{c}=\underline{\Theta}(\underline{x}, t) \quad \text { for } \underline{x} \in \partial \underline{\Omega}(t),
$$

where $\partial \underline{c} / \partial n=\underline{\hat{n}} . \nabla \underline{c}$ and $\underline{\hat{n}}$ is the unit normal to the boundary $\partial \underline{\Omega}(t)$.

In Eq. (3) we see that the effect of domain growth is to introduce two extra terms into the problem: $\underline{a} . \nabla \underline{c}$, an advection term representing the transport of material around $\underline{\Omega}(t)$ at a rate determined by the flow $\underline{a}$; and $\underline{c} \nabla \cdot \underline{a}$, a diluting (or concentrating) term due to local volume expansion (contraction) [5].

\subsection{Determination of the flow and the Lagrangian formulation}

In some cases the flow may be specified directly, see for example [4], but in the biological context under consideration here, the growth (and hence flow) rate will depend on local values of the chemical constituents reacting on the domain. Crampin and co-workers $[4,5]$ demonstrate that the Lagrangian description is particularly suitable for this purpose: it allows for the tracking of elemental volumes throughout the tissue. We choose to work with Lagrangian coordinates $(\underline{X}, t)$, such that $\underline{X}$ is the initial position of an element moving in the flow, $\underline{a}$, and use a growth function which is directly related to the Lagrangian description:

$$
\underline{x}=\underline{\Gamma}(\underline{X}, t), \quad \underline{x} \in \underline{\Omega}(t),
$$

with an initial condition given by $\underline{\Gamma}(\underline{X}, 0)=\underline{X}$. Since solid body rotations and translations do not alter growth rates we ignore them, allowing us to specify 
$\underline{\Gamma}(\underline{0}, t)=\underline{0}$. The local flow is then given by

$$
\underline{a}(\underline{X}, t)=\frac{\partial \underline{x}}{\partial t}=\frac{\partial \underline{\Gamma}}{\partial t} .
$$

Local growth The deformation due to growth can be described by the local stress tensor $\mathfrak{L}_{i j}[4,5,16]$. Here we will ignore the antisymmetric part, $\mathfrak{W}_{i j}$, of $\mathfrak{L}_{i j}$ which corresponds to rigid body rotations and as such has no effect on the chemical pattern, and concentrate on the symmetric part, $\mathfrak{D}_{i j}$, which corresponds to the rate of strain tensor. The diagonal elements represent the rate of extension along axis $x_{i}$ and the off-diagonal elements the rate of shear between axes. The rate of volume expansion in $n$ spatial dimensions is given by $[4,5]$ :

$$
\sum_{i=1}^{n} \mathfrak{D}_{i i}=\nabla \cdot \underline{a}=S(\underline{X}, t)
$$

Following [5] we take

$$
\mathfrak{D}_{i j}=\frac{\partial a_{i}}{\partial x_{j}} \Rightarrow \frac{\partial^{2} \Gamma_{i}}{\partial t \partial X_{k}}=\sum_{j=1}^{n} \frac{\partial a_{i}}{\partial x_{j}} \frac{\partial \Gamma_{j}}{\partial X_{k}}
$$

which allows calculation of $\underline{\Gamma}(\underline{X}, t)$ and $\underline{a}(\underline{X}, t)$.

\subsection{Domain growth in one spatial dimension}

In one spatial dimension the problem simplifies somewhat: we have $x=\Gamma(X, t)$ and

$$
\frac{\partial^{2} \Gamma}{\partial t \partial X}=\frac{\partial a}{\partial x} \frac{\partial \Gamma}{\partial X}=S(X, t) \frac{\partial \Gamma}{\partial X} .
$$

The original model, given by Eq. (3), reduces to the following:

$$
\begin{aligned}
\frac{\partial \underline{c}}{\partial t}+a(X(x, t), t) \frac{\partial \underline{c}}{\partial x} & =\mathcal{D} \frac{\partial^{2} \underline{c}}{\partial x^{2}}+\underline{R}(\underline{c})-S(X(x, t), t) \underline{c} \\
\frac{\partial^{2} \Gamma}{\partial t \partial X} & =S(X, t) \frac{\partial \Gamma}{\partial X} .
\end{aligned}
$$

The solution is defined for $x \in[0, l(t)]$ where $l(t)=\Gamma(l(0), t)$.

\subsection{Lagrangian coordinate system in one spatial dimension}

Inherent in the above formulation is the fact that we are able to invert $\Gamma(X, t)$ in order to calculate $a(X(x, t), t)$ and $S(X(x, t), t)$ (See Eqs. (3) and (10)). In many 
such systems this may not be possible and it may be much simpler to carry out analysis in the Lagrangian coordinate system [4,5]. Letting $(x, t) \mapsto(X, t)$ the one-dimensional system becomes

$$
\begin{aligned}
\frac{\partial \underline{c}}{\partial t} & =\mathcal{D} \frac{1}{\Gamma_{X}} \frac{\partial}{\partial X}\left(\frac{1}{\Gamma_{X}} \frac{\partial \underline{c}}{\partial X}\right)+\underline{R}(\underline{c})-S(X, t) \underline{c}, \\
\frac{\partial^{2} \Gamma}{\partial t \partial X} & =S(X, t) \frac{\partial \Gamma}{\partial X}
\end{aligned}
$$

where the solution is defined for $X \in[0, l(0)]$. Initial conditions are of the form

$$
\underline{c}(0, t)=\underline{c}_{0}, \quad \Gamma(X, 0)=X,
$$

and boundary conditions

$$
\underline{\Phi}(\underline{x}, t) \frac{\partial \underline{c}}{\partial n}+\underline{\Psi}(\underline{x}, t) \cdot \underline{c}=\underline{\Theta}(\underline{x}, t), \quad \Gamma(0, t)=0 .
$$

The solution can be recovered on the growing domain by scaling the solution along the trajectories $\Gamma(X, t)$.

\section{Initial Dpp model}

As outlined in Sect. 1.1, we will be interested in modelling the interaction of the ligand Dpp with its receptor Tkv, and we will use the observations and techniques outlined in Sects. 2 and 3 to do this. Following the experimental observations outlined in Sect. 2, we will assume that Dpp is secreted at the AP compartment boundary of the wing, whereupon it diffuses, creating a gradient in Dpp concentration along the medial-lateral axis of the wing (See Fig. 1). Dpp binds to Tkv receptors on the cell surface, and in this bound state it cannot diffuse. Here we will ignore endocytotic trafficking but assume that in its bound state (Dpp-Tkv) the receptor may be internalised and undergo decay. The association and dissociation rates of Dpp and Tkv binding are assumed to follow the Law of Mass action such that:

$$
\mathrm{Dpp}+\mathrm{Tkv} \underset{k_{-1}}{\stackrel{k_{1}}{\rightleftharpoons}} \mathrm{Dpp}-\mathrm{Tkv} .
$$

Growth of the wing results from cell proliferation, which is controlled by a combination of the concentrations of Dpp ligand and free Tkv receptors. Letting $d$ denote Dpp concentration, $r$ denote Tkv concentration and $b$ denote Dpp-Tkv concentration we propose the following model to describe Dpp and 
Tkv dynamics in the wing:

$$
\begin{aligned}
& \frac{\partial d}{\partial t}+\nabla \cdot(\underline{a} d)=\mathcal{D} \nabla^{2} d-k_{1} d r+k_{-1} b-\alpha d, \\
& \frac{\partial r}{\partial t}+\nabla \cdot(\underline{a} r)=-k_{1} d r+k_{-1} b+f(r, b, d)-g(r, b, d), \\
& \frac{\partial b}{\partial t}+\nabla \cdot(\underline{a} b)=k_{1} d r-k_{-1} b-\gamma b,
\end{aligned}
$$

where $\mathcal{D}, k_{1}, k_{-1}, \alpha$ and $\gamma$ are positive constants and $\underline{a}$ describes the flow due to growth. Both $d$ and $b$ are assumed to undergo linear decay. The terms $f$ and $g$ describe the production and decay rates of the receptor, Tkv, and possible forms for these will be suggested in Sect. 4.1. The system holds for $\underline{x} \in \underline{\Omega}(t)$ with initial conditions

$$
d(\underline{x}, 0)=d_{0}(\underline{x}), \quad r(\underline{x}, 0)=r_{0}(\underline{x}), \quad b(\underline{x}, 0)=b_{0}(\underline{x}),
$$

and boundary conditions of the form

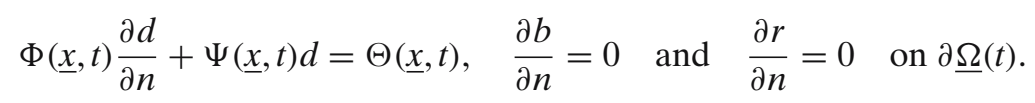

The boundary condition for $d$ is chosen such that $d$ takes a constant value, $d_{1}$, along the AP edge of the wing and satisfies zero flux boundary conditions on the remainder of the domain.

\subsection{Growth rates}

Following [21] we suppose that there are two mechanisms via which cell division (and hence wing growth) may occur. Rogulja and co-workers observe experimentally that the juxtaposition of cells which have different levels of Dpp activity is necessary and sufficient for cell proliferation in the Drosophila wing [21]. We therefore suppose that the magnitude of the gradient of Dpp must lie above a certain threshold level in order to initiate cell proliferation. This suggests that the mechanism will only promote growth in medial regions of the wing, where the gradient of Dpp activity is likely to be steeper.

The second observation made in [21] is that Dpp activity can also promote cell proliferation in a cell autonomous manner, and they suggest that this is via the Brinker pathway. They observe that the response is localised to lateral regions of the wing where the level of Dpp activity is low. We therefore suggest that cell proliferation will also be promoted by a high level of unbound Tkv receptor (as this will correspond with a low Dpp activity level).

Taking the above experimental evidence into account, we suggest the following form for the local strain rate, in $n$ spatial dimensions and in $(\underline{x}, t)$ coordinates: 


$$
S(\underline{x}, t)=\sum_{i=1}^{n} \epsilon_{d, i} H\left(-\frac{\partial d}{\partial x_{i}}(\underline{x}, t)-d^{*}\right)+\epsilon_{r, i} H\left(r(\underline{x}, t)-r^{*}\right),
$$

where $\epsilon_{d, i}, \epsilon_{r, i}$ (for $\left.i=1, \ldots, n\right), d^{*}$ and $r^{*}$ are positive constants and $H$ is the Heaviside function. In other words, we see growth only if either the gradient in Dpp is above a threshold level or if the concentration of unbound receptors is above a threshold level. These correspond to the positional information thresholds specified in Wolpert's model [28]. In one spatial dimension we can transform to Lagrangian coordinates so that:

$$
S(X, t)=\epsilon_{d} H\left(-d_{X}(X, t) / \Gamma_{X}(X, t)-d^{*}\right)+\epsilon_{r} H\left(r(X, t)-r^{*}\right) .
$$

We note here that there may be a limiting strain rate, arising from a biological constraint such as cell cycle time, for the rate of cell proliferation. In this way, if the sum of the strain rates $\epsilon_{d}+\epsilon_{r}$ is greater than this limiting rate, it would be more realistic to take some other form for $S$ (such as the maximal strain rate, for example). Although not considered here, this will be taken into account in future work and when considering biologically realistic parameters.

\subsection{Functional forms for $f$ and $g$}

We assume that $\mathrm{Tkv}$ receptor production displays an inverse relationship to the number of receptors present on a cell. Hence possible forms for $f$ are

$$
f(r, b, d)=\frac{\rho R_{\max }^{n_{1}}}{(b+r)^{n_{1}}+R_{\max }^{n_{1}}} \quad \text { and } \quad f(r, b, d)=\rho\left(1-\frac{b+r}{2 R_{\max }}\right)
$$

where $\rho, R_{\max }$ and $n_{1}$ are positive constants. In both cases $f$ is a decreasing function of $r$ and $b$. The factor of two in the second option is chosen for comparison: $b+r=R_{\max } \Rightarrow f=\rho / 2$ in each case. Plots of $f$ are shown in Fig. 2a.

It has also been shown experimentally [15] that Dpp enhances the degradation of the ligand Tkv. In this way we suggest the following forms for $g$ :

$$
g(r, b, d)=\beta r\left(1+\frac{\sigma d^{n_{2}}}{h^{n_{2}}+d^{n_{2}}}\right) \quad \text { and } \quad g(r, b, d)=\beta r\left(1+\frac{\sigma}{2 h} d\right)
$$

where $\sigma, \beta, h$ and $n_{2}$ are positive constants. In contrast, $g$ is an increasing function of $d$. We retain the factor of two to ensure that in each case $d=h \Rightarrow$ $f=\beta r(1+\sigma / 2)$. We make the short note here that it may be more biologically realistic to suppose that the bound form of Dpp enhances the degradation of Tkv: in this case $b$ should replace $d$ in Eq. (25). This will be investigated in future work, but in any case, should not make a significant difference to the model results. Plots of $g$ are shown in Fig. $2 b$. 

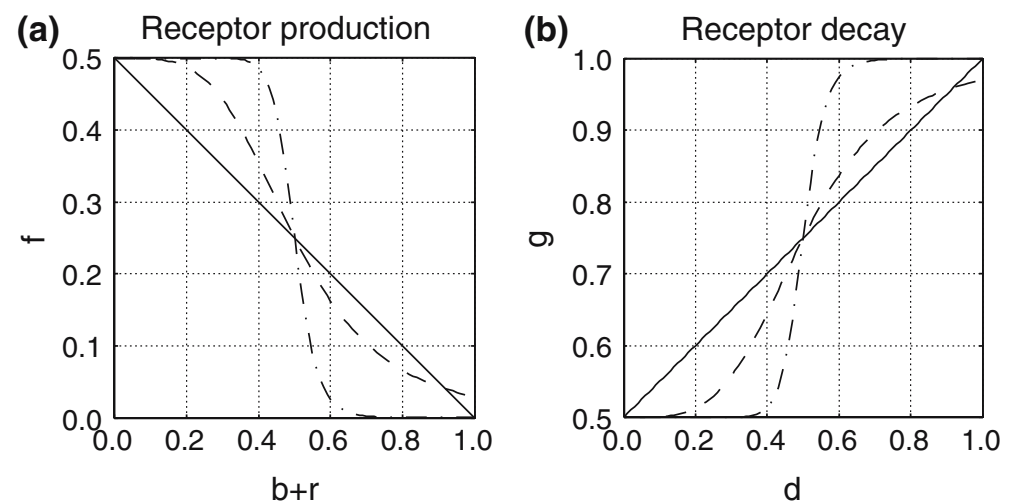

Fig. 2 a The possible forms for $f$ given in Eq. (24). The dashed line represents the first choice for $f$ with $n_{1}=4.0$, the dash-dotted line represents the first choice for $f$ with $n_{1}=16.0$ and the solid line represents the second choice for $f$. Parameters are as follows: $\rho=0.5$ and $R_{\max }=0.5$. b The possible forms for $g$ given in Eq. (25). The dashed line represents the first choice for $g$ with $n_{2}=4.0$, the dash-dotted line represents the first choice for $g$ with $n_{2}=16.0$ and the solid line represents the second choice for $g$. Parameters are as follows: $\beta=1.0, r=0.5, \sigma=1.0$ and $h=0.5$

\subsection{Reduction of the model}

The Drosophila wing disc consists of a monolayered epithelial sac of cells $[21,29]$. Dpp is secreted uniformly along the AP compartment boundary in a stripe running in a DV direction [23], See Fig. 1. We ignore the axis running perpendicular to the surface shown in Fig. 1 as the wing consists of a single layer of cells in this direction. Since Dpp is also expressed in a stripe along the DV axis, we assume that there will be little change in Dpp activity in the DV direction and so we also ignore this axis. Along the AP axis, the domain is almost symmetric about the AP compartment boundary and Dpp is secreted into both compartments. In this way we will model Tkv activity in one spatial dimension; out from the AP compartment boundary, in a posterior direction, along the AP axis. We can consider growth in the anterior compartment as mirroring growth in the posterior compartment. The region close to the AP compartment boundary is known as the medial region, and that close to the edge of the wing disc as the lateral region.

Equations (17)-(19) can therefore be written

$$
\begin{aligned}
& \frac{\partial d}{\partial t}+\frac{\partial(a d)}{\partial x}=\mathcal{D} \frac{\partial^{2} d}{\partial x^{2}}-k_{1} d r+k_{-1} b-\alpha d, \\
& \frac{\partial r}{\partial t}+\frac{\partial(a r)}{\partial x}=-k_{1} d r+k_{-1} b+f(r, b, d)-g(r, b, d), \\
& \frac{\partial b}{\partial t}+\frac{\partial(a b)}{\partial x}=k_{1} d r-k_{-1} b-\gamma b,
\end{aligned}
$$

for $x \in \Omega(t)$, where $\Omega(t)=[0, l(t)]$. The spatial axis and $l(t)$ are clearly marked in Fig. 1. 


\subsubsection{Boundary conditions}

We take boundary conditions of the form

$$
\begin{gathered}
d(0, t)=d_{1} \quad \text { and } \quad \frac{\partial r}{\partial x}=0, \frac{\partial b}{\partial x}=0 \quad \text { for } x=0 . \\
\frac{\partial d}{\partial x}=0, \quad \frac{\partial r}{\partial x}=0, \frac{\partial b}{\partial x}=0 \quad \text { for } x=l(t) .
\end{gathered}
$$

\subsubsection{Initial conditions}

We take initial conditions of the form

$$
d(x, 0)=0 \quad \text { for } x \in(0, l(t)], r(x, 0)=0, b(x, 0)=0 \quad \text { for } x \in[0, l(t)]
$$

\subsubsection{Non-dimensionalisation}

We non-dimensionalise by taking:

$$
\begin{gathered}
d=d_{1} \hat{d}, \quad r=R_{\max } \hat{r}, \quad b=R_{\max } \hat{b}, \quad x=l(0) \hat{x}, \quad t=\frac{\hat{t}}{\gamma}, \\
\hat{\mathcal{D}}=\frac{\mathcal{D}}{l(0)^{2} \gamma}, \quad \hat{\rho}=\frac{\rho}{\gamma R_{\max }}, \quad \hat{\beta}=\frac{\beta}{\gamma}, \quad \hat{\sigma}=\sigma, \quad \hat{h}=\frac{h}{d_{0}}, \\
\hat{\alpha}=\frac{\alpha}{\gamma}, \quad \hat{a}=\frac{a}{l(0) \gamma}, \quad \epsilon=\frac{R_{\max }}{d_{0}}, \quad \hat{k}_{1}=\frac{k_{1} d_{0}}{\gamma}, \quad \hat{k}_{-1}=\frac{k_{-1}}{\gamma} .
\end{gathered}
$$

Dropping the^s we arrive at the resulting system of non-dimensional equations

$$
\begin{aligned}
& \frac{\partial d}{\partial t}+\frac{\partial(a d)}{\partial x}=\mathcal{D} \frac{\partial^{2} d}{\partial x^{2}}+\epsilon\left(-k_{1} d r+k_{-1} b\right)-\alpha d, \\
& \frac{\partial r}{\partial t}+\frac{\partial(a r)}{\partial x}=-k_{1} d r+k_{-1} b+f(r, b, d)-g(r, b, d), \\
& \frac{\partial b}{\partial t}+\frac{\partial(a b)}{\partial x}=k_{1} d r-k_{-1} b-b,
\end{aligned}
$$

for $x \in \Omega(t)$, where $\Omega(t)=[0, l(t)]$ and $l(0)=1$. The functions $f$ and $g$ are now given by

$$
f(r, b, d)=\frac{\rho}{(b+r)^{n_{1}}+1} \quad \text { and } \quad f(r, b, d)=\rho\left(1-\frac{b+r}{2}\right),
$$

and

$$
g(r, b, d)=\beta r\left(1+\frac{\sigma d^{n_{2}}}{h^{n_{2}}+d^{n_{2}}}\right) \quad \text { and } \quad g(r, b, d)=\beta r\left(1+\frac{\sigma}{2 h} d\right) .
$$


The boundary conditions become

$$
\begin{gathered}
d(0, t)=1 \quad \text { and } \quad \frac{\partial r}{\partial x}=0, \quad \frac{\partial b}{\partial x}=0 \quad \text { for } x=0 . \\
\frac{\partial d}{\partial x}=0, \quad \frac{\partial r}{\partial x}=0, \quad \frac{\partial b}{\partial x}=0 \quad \text { for } x=l(t),
\end{gathered}
$$

and the initial conditions

$$
d(x, 0)=0 \quad \text { for } x \in(0, l(t)], r(x, 0)=0, b(x, 0)=0 \quad \text { for } x \in[0, l(t)] .
$$

From Eq. (22) we take $\hat{d}^{*}=d^{*} l(0) / R_{\max }$ and $\hat{r}^{*}=r^{*} / R_{\max }$. Dropping the ${ }^{\wedge}$ 's the non-dimensional strain rate in one spatial dimension then becomes

$$
S(x, t)=\epsilon_{d} H\left(-d_{x}(x, t)-d^{*}\right)+\epsilon_{r} H\left(r(x, t)-r^{*}\right),
$$

and hence

$$
S(X, t)=\epsilon_{d} H\left(-d_{X}(X, t) / \Gamma_{X}(X, t)-d^{*}\right)+\epsilon_{r} H\left(r(X, t)-r^{*}\right) .
$$

\subsection{Numerical solution}

In the system given by Eqs. (35)-(37) the local strain rate (and hence the flow rate $a$ ) is specified by Eq. (44). In this case we cannot determine $\Gamma(X, t)$ analytically and it becomes simpler to compute the numerical solution by transforming the system to Lagrangian coordinates, as discussed in Sect. 3.4. In this case we have

$$
\begin{aligned}
\frac{\partial d}{\partial t} & =\mathcal{D} \frac{1}{\Gamma_{X}} \frac{\partial}{\partial X}\left(\frac{1}{\Gamma_{X}} \frac{\partial d}{\partial X}\right)+\epsilon\left(-k_{1} d r+k_{-1} b\right)-\alpha d-S(X, t) d, \\
\frac{\partial r}{\partial t} & =-k_{1} d r+k_{-1} b+f(r, b, d)-g(r, b, d)-S(X, t) r, \\
\frac{\partial b}{\partial t} & =k_{1} d r-k_{-1} b-b-S(X, t) b, \\
\frac{\partial}{\partial t}\left(\frac{\partial \Gamma}{\partial X}\right) & =S(X, t) \frac{\partial \Gamma}{\partial X},
\end{aligned}
$$

where $S(X, t)$ is given by Eq. (44).

The above system can be solved by converting to a system of first order PDEs in $d, d_{X}, r, b, \Gamma$ and $\Gamma_{X}$ and employing the NAG routine D03PEF. The routine uses a Keller box scheme for the spatial discretisation and the resulting system of equations is solved using a Backward Differentiation Formula method [20]. The solutions may be transformed onto the growing domain using the MATLAB function interp. 


\subsection{Results}

Numerical approximations to the system for the first suggested forms of $f$ and $g$ can be seen in Fig. 3 and for the second forms in Fig. 4: we note that results of the numerical computations are similar for both forms of $f$ and $g$. A Dpp gradient is maintained across the wing disc: high in the medial region close to the source, and decreasing in a lateral direction. The Dpp gradient is mirrored
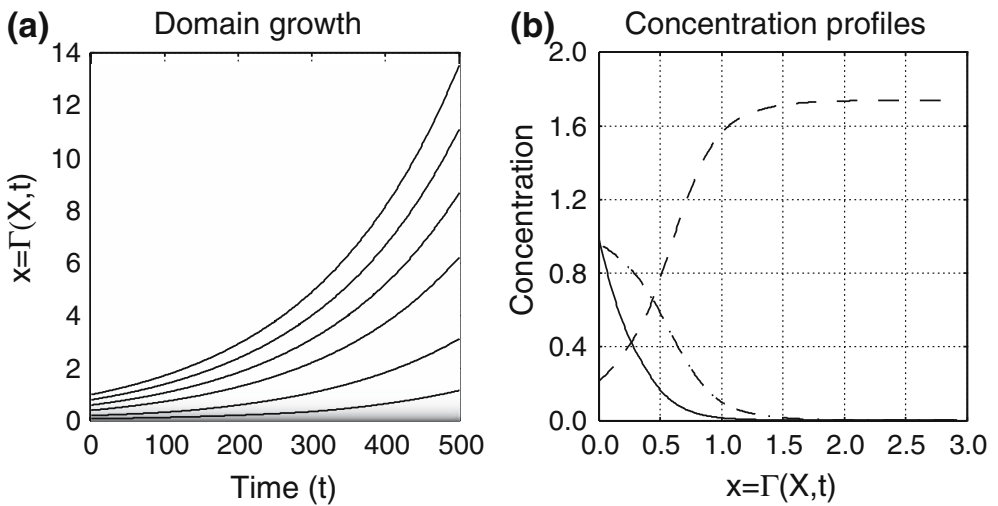

Fig. 3 Numerical solutions for the Dpp-Tkv model as given by Eqs. (44) and (45)-(48). Here we use the first forms for $f$ and $g$ from Eqs. (38) and (39). a Plot of the trajectories, $\Gamma(X, t)$, along with the Dpp gradient. The initial points of the trajectories are evenly spaced along the interval $[0,1]$. b Concentration profiles at time $t=200$. Solid line - $d$; Dashed line - $r$; Dash-dotted line b. Parameters are as follows: $D=1.0, k_{1}=5.0, k_{-1}=0.1, \epsilon=1.0, \alpha=10.0, \rho=0.8, \beta=0.5$, $\sigma=10.0, h=20, d^{*}=0.8, r^{*}=0.5, \epsilon_{d}=0.005, \epsilon_{r}=0.005, n_{1}=4.0$ and $n_{2}=4.0$
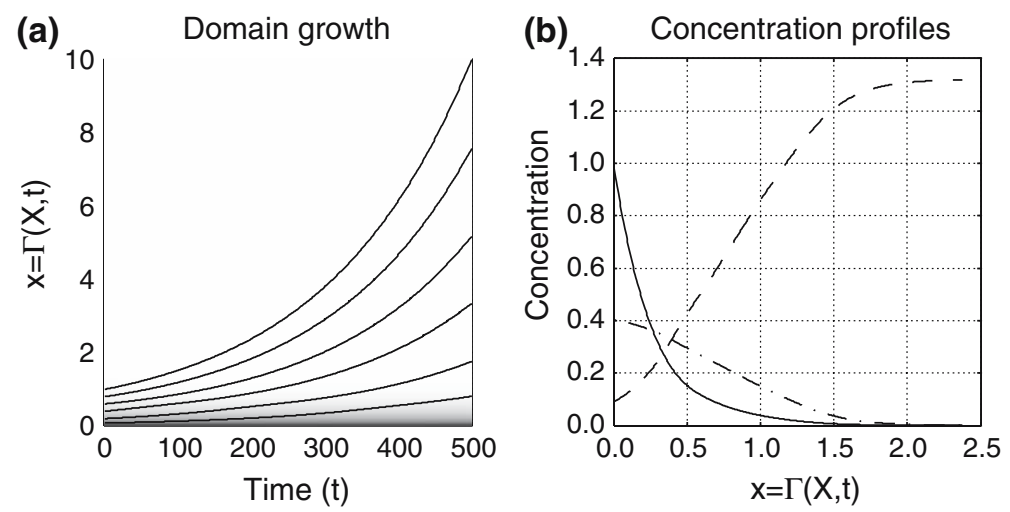

Fig. 4 Numerical solutions for the Dpp-Tkv model as given by Eqs. (44) and (45)-(48). Here we use the second forms for $f$ and $g$ from Eqs. (38) and (39). a Plot of the trajectories, $\Gamma(X, t)$, along with the Dpp gradient. The initial points of the trajectories are evenly spaced along the interval $[0,1]$. b Concentration profiles at time $t=200$. Solid line - $d ;$ Dashed line - $r$; Dash-dotted line b. Parameters are as follows: $D=1.0, k_{1}=5.0, k_{-1}=0.1, \epsilon=1.0, \alpha=10.0, \rho=0.8, \beta=0.2$, $\sigma=10.0, h=0.5, d^{*}=0.8, r^{*}=0.5, \epsilon_{d}=0.005$ and $\epsilon_{r}=0.005$ 

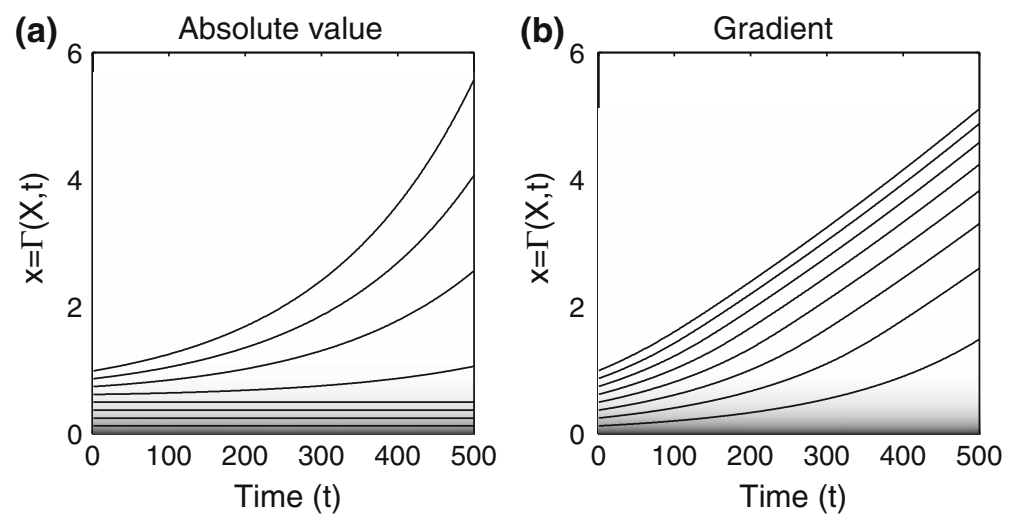

Fig. 5 Numerical solutions for the Dpp-Tkv model as given by Eqs. (44) and (45)-(48). a Growth due to the absolute level of Dpp. The bottom three trajectories remain horizontal indicating zero growth in a region close to $x=0$. The curvature of the fourth trajectory indicates that the boundary of the growth region lies approximately between $x=0.5$ and $x=0.625$. In this case $\epsilon_{d}=0.0$. b Growth due to the gradient in Dpp. A linear trajectory indicates a region in which local domain growth is not occurring. In this case we see that growth occurs initially throughout the domain, but as the domain expands lateral areas leave the growing region. Here $\epsilon_{r}=0.0$. In both cases the initial points of the trajectories are evenly spaced along the interval $[0,1]$. Here we use the first forms for $f$ and $g$ from Eqs. (38) and (39). Unless otherwise stated parameters are as follows: $D=1.0, k_{1}=5.0, k_{-1}=0.1, \epsilon=1.0, \alpha=10.0, \rho=0.8, \beta=0.2, \sigma=10.0, h=0.5, d^{*}=0.01$, $r^{*}=0.9, \epsilon_{d}=0.005$ and $\epsilon_{r}=0.005$

by the number of bound Tkv receptors and opposed by a gradient of unbound Tkv receptors, as observed experimentally.

Figure 5 shows numerical approximations of the model, with growth due to the absolute level and growth due to the gradient considered separately. Figure 5a demonstrates domain growth arising with growth due to the level of Dpp. The bottom three trajectories are horizontal showing the absence of growth in a region close to $x=0$. Figure $5 \mathrm{~b}$ demonstrates domain growth arising due to the gradient of Dpp activity. The upper trajectories quickly become parallel to one another indicating that lateral regions of the disc quickly move out of the region where growth occurs, whilst regions initially lying close to $x=0$ continue to expand over a much longer time period.

Inherent in our construction of the model is the assumption that growth, when it does occur, is regionally uniform at either rate $0, \epsilon_{d}, \epsilon_{r}$ or $\epsilon_{d}+\epsilon_{r}$, depending on the region of the wing in which a cell is sitting. Experimental observations suggest that domain growth is uniform across the wing disc [21] and in turn, this suggests that growth due to the gradient in Dpp must occur at a similar rate to growth due to the level of Tkv receptors. Hence in our model we have assumed that the parameters $\epsilon_{d}$ and $\epsilon_{r}$ have similar magnitudes. We also note here that, at present, none of our parameter choices are informed by experimental data. One objective for the future is to obtain, as a far as possible, experimentally measured parameter values.

Numerical simulations of the model suggest that such growth results in an exponentially increasing domain size. One observation that should be noted is 
that if there is no growth due to the level of unbound Tkv receptors, only due to the Dpp gradient, then after an initial period, the global (rather than local) growth of the disc occurs at a uniform rate: cells proliferate and are displaced (due to growth) at an exponential rate until they reach the region where the Dpp gradient is no longer steep enough to stimulate proliferation. The result is that the lateral boundary of the wing moves at a constant rate. At this point, a trajectory becomes a straight line in $(x, t)$-space, parallel to the trajectories preceding it: this is an indication of zero growth in a region. We also note that uniform growth across the disc will obviously only be achieved if there is a near correlation in the boundaries of the regions in which growth due to each mechanism occurs. We will discuss these matters further in Sect. 5.

\section{Simplification of the model}

Although the model developed in the Sect. 4 displays the behaviour observed in vivo for the parameter values listed in Figs. 3 and 4, and for a range of other values not shown here, the system is not particularly amenable to analytical exploration and as such it is difficult to gain further insight into the growth processes involved. In the absence of any approximate parameter values/ratios with which to simplify the model, we make some heuristic approximations (See Sect. 6 for more discussion of these matters). Firstly, we note that the gradient in Dpp decays exponentially across the wing disc, that this gradient is mirrored by a gradient in bound receptor Dpp-Tkv, and is opposite to the gradient of unbound receptor, Tkv. Therefore we propose a model in which a Dpp concentration gradient regulates both types of growth, i.e. where the slope of the Dpp gradient regulates growth in medial regions of the disc and the absolute value of Dpp regulates growth laterally.

In order to do this, we consider the evolution of a single chemical, $c$, on a one-dimensional, growing domain in which growth is controlled by $c$ itself. In line with the previous system, we suppose that $c$ is secreted at the boundary of the domain given by $x=0$ and that within the domain, it diffuses and undergoes linear decay. We consider the following non-dimensional evolution equation for $c$ :

$$
\frac{\partial c}{\partial t}+\frac{\partial(a c)}{\partial x}=\mathcal{D} \frac{\partial^{2} c}{\partial x^{2}}-\lambda c,
$$

for $x \in[0, l(t)]$, with boundary conditions: $c(0, t)=1$ and $\partial c / \partial x(1, t)=0$. The initial conditions are $l(0)=1$ and $c(x, 0)=c_{0}(x) . c_{0}(x)$ is found by considering the steady state gradient formed on a static domain of length $l(0)=1$ :

$$
c_{0}(x)=\frac{1}{e^{\beta}+e^{-\beta}}\left[e^{\beta(x-1)}+e^{-\beta(x-1)}\right], \quad x \in[0,1],
$$

where $\beta=\sqrt{\lambda / \mathcal{D}}$. 
Here the local rate of volume expansion is given in $(x, t)$ coordinates by

$$
S(x, t)=\alpha_{g} H\left(-c_{x}(x, t)-c_{g}\right)+\alpha_{a} H\left(1-c(x, t)-c_{a}\right),
$$

i.e. growth occurs in the medial region (close to the AP compartment boundary) due to a gradient in the chemical above a threshold, $c_{g}$, and in the lateral region (away from the AP compartment boundary) due to the chemical falling below a threshold value, $1-c_{a}$. The former corresponds to growth due to a gradient in $d$ above the threshold and the latter due to $r$ levels remaining above the threshold.

In Lagrangian coordinates, we have

$$
\begin{aligned}
\frac{\partial c}{\partial t} & =\mathcal{D} \frac{1}{\Gamma_{X}} \frac{\partial}{\partial X}\left(\frac{1}{\Gamma_{X}} \frac{\partial c}{\partial X}\right)-\lambda c-S(X, t) c \\
\frac{\partial}{\partial t}\left(\frac{\partial \Gamma}{\partial X}\right) & =S(X, t) \frac{\partial \Gamma}{\partial X}
\end{aligned}
$$

where

$$
S(X, t)=\alpha_{g} H\left(-c_{X}(X, t) / \Gamma_{X}-c_{g}\right)+\alpha_{a} H\left(1-c(X, t)-c_{a}\right),
$$

and $X \in[0,1]$.

The numerical approximation can be found, as before, by employing the NAG routine D03PEF and the results are shown in Fig. 6. The plots show similar results to the full model: a chemical gradient across the domain results in exponential growth of the domain. Although variation of $c_{g}$ and $c_{a}$ can result in regions of the disc in which there is no growth, the trajectories in Fig. 6 indicate that in this case growth occurs across most of the disc.
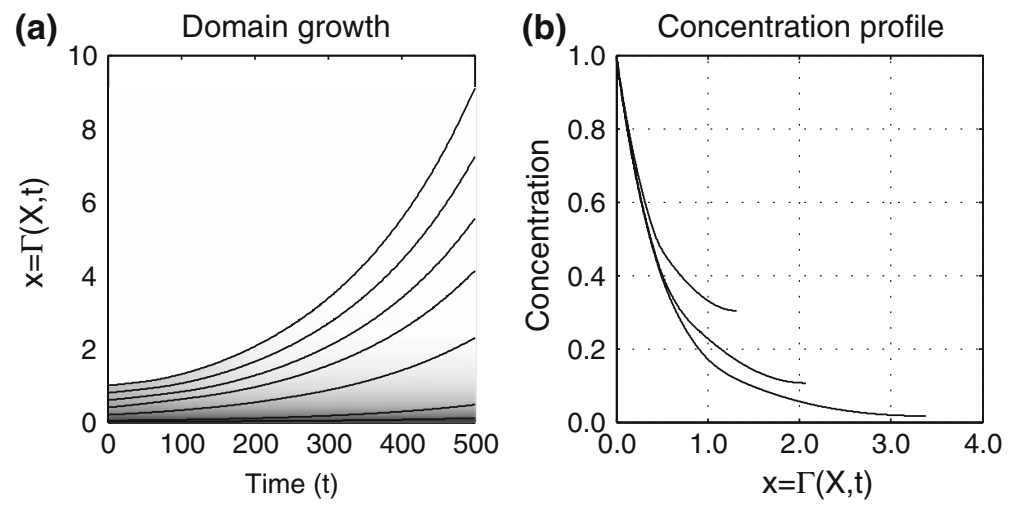

Fig. 6 Numerical solution for the reduced model as given by Eqs. (52), (53) and (54). a Trajectories and the chemical gradient $(c)$. b The chemical profile at times $t=100,200,300$. Parameters are as follows: $D=10.0, \lambda=20, \alpha_{a}=0.005, \alpha_{g}=0.005, c_{a}=0.6$ and $c_{g}=0.6$ 


\subsection{Limiting case}

As the domain continues to grow, and in the case where transport and dilution of the chemical due to growth do not play a major role in shaping the gradient, we expect that after a certain time the gradient will reach an approximately steady state. This can be verified by numerical computation (not shown here) and we will use this in order to facilitate analysis of the model.

The limiting case described above can be considered mathematically by assuming that $\alpha_{a}, \alpha_{g}, \beta \ll 1$. In this case we may assume that $a(X, t), S(X, t) \ll 1$ and, from Eq. (50), that the chemical concentration is in a steady state which is given approximately by

$$
c(x, t)=e^{-\beta x} .
$$

In order to calculate the rate of domain growth, we must therefore solve the equation

$$
\frac{\partial}{\partial t}\left(\frac{\partial \Gamma}{\partial X}\right)=S(X, t) \frac{\partial \Gamma}{\partial X}
$$

with

$$
S(X, t)=\alpha_{g} H\left(\beta e^{-\beta \Gamma}-c_{g}\right)+\alpha_{a} H\left(1-e^{-\beta \Gamma}-c_{a}\right),
$$

and initial and boundary conditions $\Gamma(X, 0)=X$ and $\Gamma(0, t)=0$, respectively.

We first note that there are three regions, each with different growth rates, and that we must solve in each region separately (See Fig. 7). Taking

$$
x_{g}=\frac{1}{\beta} \log \left(\frac{\beta}{c_{g}}\right) \quad \text { and } \quad x_{a}=\frac{1}{\beta} \log \left(\frac{1}{1-c_{a}}\right),
$$

(a) No overlap

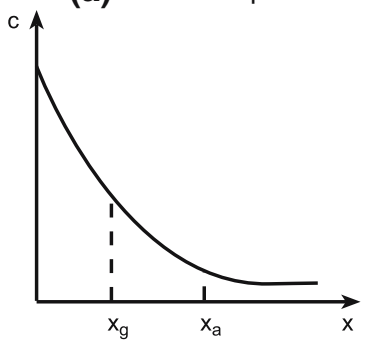

(b) Perfect Join

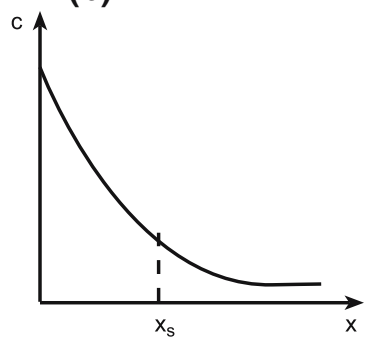

(c) With overlap

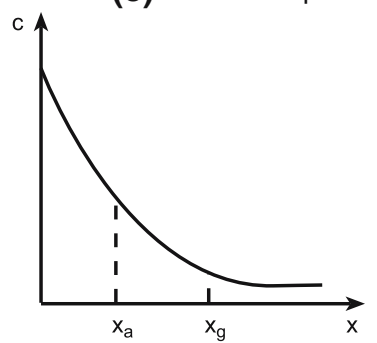

Fig. 7 The different possible regions of growth in the reduced model. a No overlap: growth occurs in the region $x<x_{g}$ at rate $\alpha_{g}$ and in the region $x>x_{a}$ at rate $\alpha_{a}$. b Perfect join: growth occurs in the region $x<x_{s}$ at rate $\alpha_{g}$ and in the region $x>x_{s}$ at rate $\alpha_{a}$. c With overlap: growth occurs in the region $x<x_{a}$ at rate $\alpha_{g}$, in the region $x_{a}<x<x_{g}$ at rate $\alpha_{a}+\alpha_{g}$ and in the region $x>x_{g}$ at rate $\alpha_{a}$ 
then $x_{g}$ represents the point at which the gradient drops below the threshold level, $c_{g}$, needed for domain growth at rate $\alpha_{g}$ and $x_{a}$ represents the point at which $c$ drops below the threshold level, $1-c_{a}$, required for domain growth at rate $\alpha_{a}$. As shown in Fig. 7, there are three different possibilities for the model (depending on the values of $c_{a}$ and $c_{g}$ ) and we discuss each below.

\subsubsection{Case I}

Initially we will assume that $x_{g}<x_{a}$ as in Fig. 7a: in which case we have the following

$$
\frac{\partial}{\partial t}\left(\frac{\partial \Gamma}{\partial X}\right)= \begin{cases}\alpha_{g} \frac{\partial \Gamma}{\partial X} & \text { for } \Gamma(X, t)<x_{g} \\ 0 & \text { for } x_{g}<\Gamma(X, t)<x_{a} \\ \alpha_{a} \frac{\partial \Gamma}{\partial X} & \text { for } \Gamma(X, t)>x_{a}\end{cases}
$$

Initially $X<x_{g}$ : Whilst $\Gamma(X, 0)=X<x_{g}$

$$
\frac{\partial}{\partial t}\left(\frac{\partial \Gamma}{\partial X}\right)=\alpha_{g} \frac{\partial \Gamma}{\partial X},
$$

with $\Gamma(X, 0)=X$ and $\Gamma(0, t)=0$. This equation can be solved trivially to give

$$
\Gamma(X, t)=X e^{\alpha_{g} t},
$$

which holds until $\Gamma(X, t)=x_{g}$, at time

$$
t=t_{g}(X)=\frac{1}{\alpha_{g}} \log \left(\frac{x_{g}}{X}\right) .
$$

Whilst $x_{g}<\Gamma(X, t)<x_{a}$,

$$
\frac{\partial}{\partial t}\left(\frac{\partial \Gamma}{\partial X}\right)=0
$$

with $\Gamma\left(X, t_{g}(X)\right)=x_{g}$. However, this equation as it stands cannot be solved uniquely (as for the region $X<x_{g}$ ) since we cannot specify a boundary condition. Instead we appeal to a solution from 'first principles': suppose we divide the spatial region $\left[0, x_{g}\right]$ into $N$ intervals of width $\delta x$ and the temporal region $\left[t_{g}, t\right]$ into $i$ intervals of width $\delta t$. Over the first time step $\delta t$ each region $\delta x$ expands to fill a region of width $\delta x\left(1+\alpha_{g} \delta t\right)$. Summing over all the regions $\delta x$ we see that at time $t=t_{g}+\delta t$ the point $x_{g}$ has moved to $x^{\prime}=x_{g}\left(1+\alpha_{g} \delta t\right)$. Applying this reasoning repeatedly we see the following mappings for the point $x_{g}$ over successive time steps $\delta t$ : 


$$
\begin{aligned}
x_{g} & \mapsto x^{\prime}=x_{g}\left(1+\alpha_{g} \delta t\right), \\
x^{\prime} & \mapsto x^{\prime \prime}=x_{g}\left[1+\alpha_{g} \delta t+\alpha_{g} \delta t\right], \\
\vdots & \vdots \\
x^{i-1} & \mapsto x^{i}=x_{g}\left[1+i \alpha_{g} \delta t\right],
\end{aligned}
$$

where $x^{k}$ indicates the position of the point $x_{g}$ after $k$ time steps of length $\delta t$. Noting $i \delta t=t-t_{g}(X)$ and taking the limit as $\delta t \rightarrow 0$ we see that

$$
\Gamma(X, t)=x_{g}+\alpha_{g} x_{g}\left[t-t_{g}(X)\right] .
$$

This holds until $\Gamma(X, t)=x_{a}$, at time

$$
t=t_{a}(X)=t_{g}(X)+\frac{1}{\alpha_{g} x_{g}}\left(x_{a}-x_{g}\right) .
$$

For $\Gamma(X, t)>x_{a}$,

$$
\frac{\partial}{\partial t}\left(\frac{\partial \Gamma}{\partial X}\right)=\alpha_{a} \frac{\partial \Gamma}{\partial X}
$$

with $\Gamma\left(X, t_{a}(X)\right)=x_{a}$. Once again, we cannot specify a boundary condition and so we derive a solution from 'first principles': initially dividing the temporal region $\left[t_{a}, t\right]$ up into $i$ intervals of width $\delta t$. Using the previous result we see that over the first time step $\delta t, x_{a}$ is mapped to $x^{\prime}=x_{a}+\alpha_{g} x_{g} \delta t$.

For the next time step $\delta t$ we divide the spatial region $\left[x_{a}, x^{\prime}\right]$ up into $N$ intervals of width $\delta x$ and note that each expands to width $\delta x\left(1+\alpha_{a} \delta t\right)$. In this way, we see that

$$
x^{\prime} \mapsto x^{\prime \prime}=\alpha_{a}+\alpha_{g} x_{g} \delta t+\left(x^{\prime}-x_{a}\right)\left(1+\alpha_{a} \delta t\right) .
$$

This is shown in Fig. 8. Continuing in this manner, we have the following mappings:

$$
\begin{aligned}
x_{a} & \mapsto x^{\prime}=x_{a}+\alpha_{g} x_{g} \delta t, \\
x^{\prime} & \mapsto x^{\prime \prime}=x_{a}+\alpha_{g} x_{g} \delta t+\alpha_{g} x_{g} \delta t\left(1+\alpha_{a} \delta t\right), \\
\vdots & \vdots \\
x^{i-1} & \mapsto x^{i}=x_{a}+\alpha_{g} x_{g} \delta t \sum_{k=0}^{i-1}\left(1+\alpha_{a} \delta t\right)^{k} .
\end{aligned}
$$

The summation can easily be evaluated to give

$$
\Gamma(X, t)=\lim _{\delta t \rightarrow 0}\left\{x_{a}+\frac{\alpha_{g} x_{g}}{\alpha_{a}}\left[\left(1+\alpha_{a} \delta t\right)^{\left(t-t_{a}(X)\right) / \delta t}-1\right]\right\},
$$


Fig. 8 The solution from 'first principles'. Over the first time step $\delta t$, the point $x_{a}$ is mapped to $x_{a}+\alpha_{g} x_{g} \delta t$. The region $\left[x^{\prime}, x_{a}\right]$ is then divided into $N$ intervals of width $\delta x$ : over the time step $\delta t$ each one grows to fill a region of width $\delta x\left(1+\alpha_{a} \delta t\right)$ etc

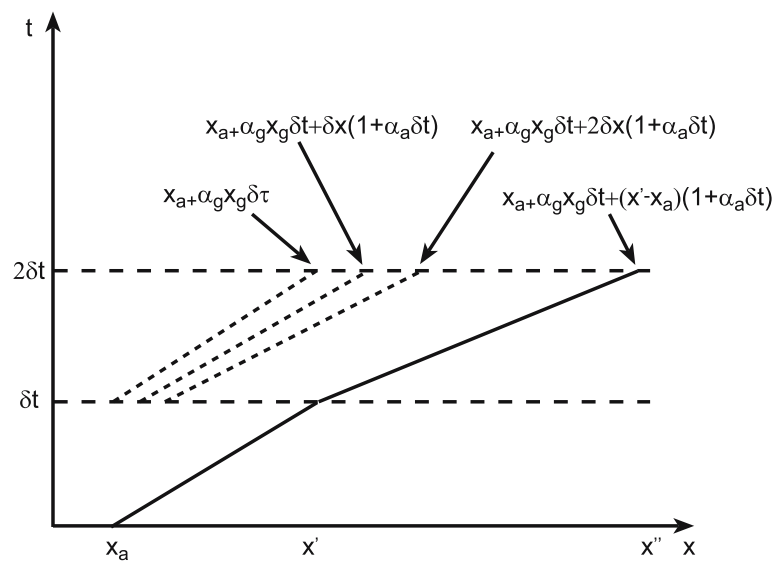

from which it can be shown that

$$
\Gamma(X, t)=x_{a}+\frac{\alpha_{g} x_{g}}{\alpha_{a}}\left[e^{\alpha_{a}\left(t-t_{a}(X)\right)}-1\right] .
$$

Initially $x_{g}<X<x_{a}$ : Whilst $\Gamma(X, t)<x_{a}$ we follow along the same lines as previously to get

$$
\Gamma(X, t)=X+\alpha_{g} x_{g} t .
$$

This holds until $\Gamma(X, t)=x_{a}$, at time

$$
t=t_{a}(X)=\frac{1}{\alpha_{g} x_{g}}\left(x_{a}-X\right) .
$$

For $\Gamma(X, t)>x_{a}$

$$
\frac{\partial}{\partial t}\left(\frac{\partial \Gamma}{\partial X}\right)=\alpha_{a} \frac{\partial \Gamma}{\partial X},
$$

with $\Gamma\left(X, t_{a}(X)\right)=x_{a}$ and, as previously, we can solve to get

$$
\Gamma(X, t)=x_{a}+\frac{\alpha_{g} x_{g}}{\alpha_{a}}\left[e^{\alpha_{a}\left(t-t_{a}(X)\right)}-1\right] .
$$

Initially $X>x_{a}:$ For all $t>0, \Gamma(X, t)$ satisfies the equation

$$
\frac{\partial}{\partial t}\left(\frac{\partial \Gamma}{\partial X}\right)=\alpha_{a} \frac{\partial \Gamma}{\partial X},
$$

with $\Gamma(X, 0)=X$. We divide the spatial interval $\left[x_{a}, X\right]$ into $N$ intervals of width $\delta x$ and the temporal interval $[0, t]$ into $i$ intervals of width $\delta t$. Over the 
first time step $\delta t$ each interval $\delta x$ expands to width $\delta x\left(1+\alpha_{a} \delta t\right)$. Summing over all the intervals $\delta x$ we see that after time $\delta t$ the point $X$ has moved to $x^{\prime}=x_{a}+\alpha_{g} x_{g} \delta t+\left(x-x_{a}\right)\left(1+\alpha_{a} \delta t\right)$. Applying this reasoning repeatedly to intervals $\left[x_{a}, x^{\prime}\right],\left[x_{a}, x^{\prime \prime}\right]$ etc. we see the following mappings for the point $X$ :

$$
\begin{aligned}
X & \mapsto x^{\prime}=x_{a}+\alpha_{g} x_{g} \delta t+\left(x-x_{a}\right)\left(1+\alpha_{a} \delta t\right), \\
x^{\prime} & \mapsto x^{\prime \prime}=x_{a}+\alpha_{g} x_{g} \delta t+\alpha_{g} x_{g} \delta t\left(1+\alpha_{a} \delta t\right)+\left(x-x_{a}\right)\left(1+\alpha_{a} \delta t\right)^{2}, \\
\vdots & \quad \vdots \\
x^{i-1} & \mapsto x^{i}=x_{a}+\alpha_{g} x_{g} \delta t \sum_{k=0}^{i-1}\left(1+\alpha_{a} \delta t\right)^{k}+\left(x-x_{a}\right)\left(1+\alpha_{a} \delta t\right)^{i} .
\end{aligned}
$$

As before, we evaluate the summation and take the limit as $\delta t \rightarrow 0$ to get the result

$$
\Gamma(X, t)=x_{a}+\frac{\alpha_{g} x_{g}}{\alpha_{a}}\left[e^{\alpha_{a} t}-1\right]+\left(x-x_{a}\right) e^{\alpha_{a} t} .
$$

The results from these calculations are summarised in Table 1 and presented graphically in Fig. 9. Figure 9a shows the trajectories given by the numerical approximation whilst Fig. 9b shows the error between the analytical solution (Table 1) and the numerical approximation to the system (Eqs. (52)-(54)). The error is negative as the analytical approximation tends to over predict growth: this will be discussed briefly in Sect. 5.2. The domain boundary is given by taking $\Gamma(X, 0)=\Gamma(1,0)$ in Table 1 and hence we see that the domain length changes according to the equation

$$
l(t)=\Gamma(1, t)=x_{a}+\frac{\alpha_{g} x_{g}}{\alpha_{a}}\left[e^{\alpha_{a} t}-1\right]+\left(1-x_{a}\right) e^{\alpha_{a} t} .
$$

As predicted by earlier analysis, the domain length increases at an exponential rate. We can also demonstrate what happens in the case that there is no cell

Table 1 Solution in the approximate case, given by Eqs. (55), (56) and (57), where $x_{g}<x_{a}$

\begin{tabular}{lll}
\hline Spatial region & Temporal region & Solution \\
\hline $0 \leq \Gamma(X, 0) \leq x_{g}$ & $0 \leq t<t_{g}(X)$ & $\Gamma(X, t)=X \mathrm{e}^{\alpha_{g} t}$ \\
& $t_{g}(X) \leq t<t_{a}(X)$ & $\Gamma(X, t)=x_{g}+\alpha_{g} x_{g}\left[t-t_{g}(X)\right]$ \\
& $t \geq t_{a}(X)$ & $\Gamma(X, t)=x_{a}+\frac{\alpha_{g} x_{g}}{\alpha_{a}}\left[\mathrm{e}^{\alpha_{a}\left(t-t_{a}(X)\right)}-1\right]$ \\
& $t_{g}(X)=\frac{1}{\alpha_{g}} \log \left(\frac{x_{g}}{X}\right)$ and $t_{a}(X)=t_{g}(X)+\frac{1}{\alpha_{g} x_{g}}\left(x_{a}-x_{g}\right)$ \\
$x_{g}<\Gamma(X, 0) \leq x_{a}$ & $0 \leq t<t_{a}(X)$ & $\Gamma(X, t)=X+\alpha_{g} x_{g} t$ \\
& $t \geq t_{a}(X)$ & $\Gamma(X, t)=x_{a}+\frac{\alpha_{g} x_{g}}{\alpha_{a}}\left[\mathrm{e}^{\alpha_{a}\left(t-t_{a}(X)\right)}-1\right]$ \\
& $t=t_{a}(X)=\frac{1}{\alpha_{g} x_{g}}\left(x_{a}-X\right)$ & $\Gamma(X, t)=x_{a}+\frac{\alpha_{g} x_{g}}{\alpha_{a}}\left[\mathrm{e}^{\alpha_{a} t}-1\right]+\left(X-x_{a}\right) \mathrm{e}^{\alpha_{a} t}$ \\
\hline$(X, 0)>x_{a}$ & $t_{a}(x) \leq t$ &
\end{tabular}



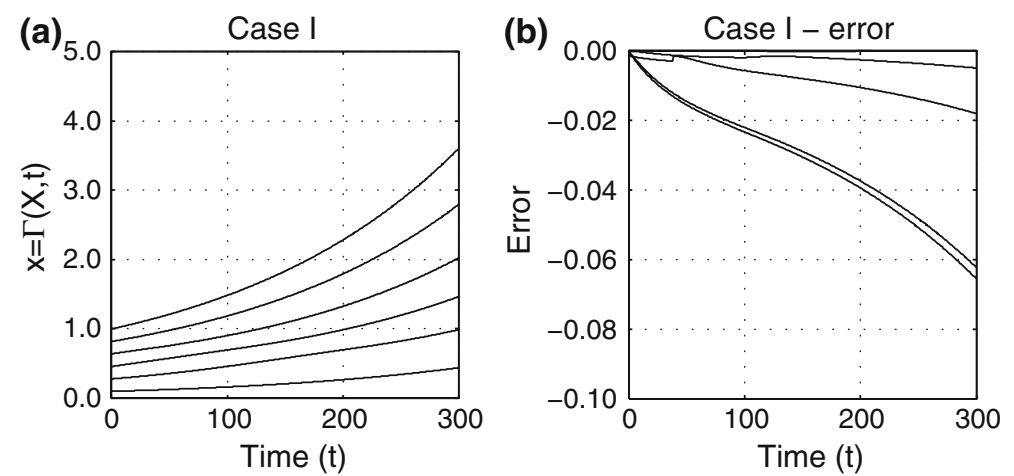

Fig. 9 Analytical approximation (see Table 1 and Eqs. (55), (56) and (57)) for the reduced model as given by Eqs. (52), (53) and (54). a Trajectories given by the approximation. $\mathbf{b}$ The deviation of the approximation from the numerical solution (the error increases as $\Gamma(X, 0)$ increases). We have plotted the numerically computed solution minus the analytical approximation. Parameters are as follows: $D=10.0, \lambda=100, \alpha_{a}=0.005, \alpha_{g}=0.005, c_{a}=0.9$ and $c_{g}=0.7$

Table 2 Solution in the approximate case, given by Eqs. (55), (56) and (57), where $x_{g}=x_{s}=x_{a}$

\begin{tabular}{lll}
\hline Spatial region & Temporal region & Solution \\
\hline $0 \leq \Gamma(X, 0) \leq x_{S}$ & $0 \leq t<t_{g}(X)$ & $\Gamma(X, t)=X \mathrm{e}^{\alpha_{g} t}$ \\
& $t \geq t_{S}(X)$ & $\Gamma(X, t)=x_{S}+\frac{\alpha_{g} x_{s}}{\alpha_{a}}\left[\mathrm{e}^{\alpha_{a}\left(t-t_{S}(X)\right)}-1\right]$ \\
& $t_{S}(X)=\frac{1}{\alpha_{g}} \log \left(\frac{x_{S}}{X}\right)$ & \\
$\Gamma(X, 0)>x_{S}$ & $t \geq 0$ & $\Gamma(X, t)=x_{S}+\frac{\alpha_{g} x_{S}}{\alpha_{a}}\left[\mathrm{e}^{\alpha_{a} t}-1\right]+\left(X-x_{a}\right) \mathrm{e}^{\alpha_{a} t}$ \\
\hline
\end{tabular}

proliferation via the brinker pathway: we may just assume that $l(t)<x_{a}, \forall t$ in which case we have:

$$
\Gamma(X, t)=x_{g}+\alpha_{g} x_{g} t
$$

and we see that the domain grows linearly. This can also be verified using numerical computations (not shown here).

\subsubsection{Cases II and III}

The remaining cases complete the picture and can be dealt with in the same manner as Case I. In Case II, Fig. 7b, $c_{g}=\beta\left(1-c_{a}\right)$ so that $x_{a}=x_{s}=x_{g}$ and the regions match exactly. The results for Case II are summarised in Table 2. In Case III, Fig. 7(c), we have $x_{a}<x_{g}$ so that there exists a region in which growth due to both the gradient and the level of $c$ is occurs; these results are summarised in Table 3. In both cases the error between the analytical approximations (Tables 2 and 3) and the numerical approximations of the system (Eqs. (52)-(54)) remain small (less marked than for Case 1). 
Table 3 Solution in the approximate case, given by Eqs. (55), (56) and (57), where $x_{a}<x_{g}$

\begin{tabular}{lll}
\hline Spatial region & Temporal region & Solution \\
\hline $0 \leq \Gamma(X, 0) \leq x_{a}$ & $0 \leq t<t_{a}(X)$ & $\Gamma(X, t)=X \mathrm{e}^{\alpha_{g} t}$ \\
& $t_{a}(X) \leq t<t_{g}(X)$ & $\Gamma(X, t)=x_{a}+\frac{\alpha_{g} x_{a}}{\alpha_{a}+\alpha_{g}}\left[\mathrm{e}^{\left(\alpha_{a}+\alpha_{g}\right)\left(t-t_{a}(X)\right)}-1\right]$ \\
& $t \geq t_{g}(X)$ & $\Gamma(X, t)=x_{g}+\frac{1}{\alpha_{a}}\left[\left(\alpha_{a}+\alpha_{g}\right) x_{g}-\alpha_{a} x_{a}\right]\left[\mathrm{e}^{\alpha_{a}\left(t-t_{g}(X)\right)}-1\right]$ \\
& $t_{a}(X)=\frac{1}{\alpha_{g} x_{g}} \log \left(\frac{x_{a}}{X}\right)$ and $t_{g}(X)=t_{a}(X)+\frac{1}{\alpha_{a}+\alpha_{g}} \log \left[\frac{\alpha_{a}+\alpha_{g}}{\alpha_{g} x_{a}}\left(x_{g}-x_{a}\right)+1\right]$ \\
$x_{a}<\Gamma(X, 0) \leq x_{g}$ & $0 \leq t<t_{g}(X)$ & $x_{a}+\frac{\alpha_{g} x_{a}}{\alpha_{a}+\alpha_{g}}\left[\mathrm{e}^{\left(\alpha_{a}+\alpha_{g}\right) t}-1\right]+\left(X-x_{a}\right) \mathrm{e}^{\left(\alpha_{a}+\alpha_{g}\right) t}$ \\
& $t \geq t_{g}(X)$ & $\Gamma(X, t)=x_{g}+\frac{1}{\alpha_{a}}\left[\left(\alpha_{a}+\alpha_{g}\right) x_{g}-\alpha_{a} x_{a}\right]\left[\mathrm{e}^{\alpha_{a}\left(t-t_{g}(X)\right)}-1\right]$ \\
& $t=t_{g}(X)=\frac{1}{\alpha_{a}+\alpha_{g}} \log \left[\frac{\left(x_{g}-x_{a}\right)\left(\alpha_{a}+\alpha_{g}\right)+\alpha_{g} x_{a}}{\left(X-x_{a}\right)\left(\alpha_{a}+\alpha_{g}\right)+\alpha_{g} x_{a}}\right]$ \\
& $t \geq 0$ & $\Gamma(X, t)=x_{g}+\frac{1}{\alpha_{a}}\left[\left(\alpha_{a}+\alpha_{g}\right) x_{g}-\alpha_{a} x_{a}\right]\left[\mathrm{e}^{\alpha_{a} t}-1\right]$ \\
$\Gamma(X, 0)>x_{a}$ & & $+\left(X-x_{g}\right) \mathrm{e}^{\alpha_{a} t}$ \\
& &
\end{tabular}

\subsection{Results}

Our analysis of the reduced model allowed us find an analytical expression for the path of cells through the domain and also to track the domain boundary. The results of this analysis show that (under certain parameter conditions) after an initial time period in which the chemical gradient reaches a 'steady state', we expect growth of the domain to be exponential at a rate dependent on each of the parameters $x_{g}, x_{a}, \alpha_{g}$ and $\alpha_{a}$. We can also confirm that if there is only growth due to the slope of the Dpp gradient then domain growth will in fact be linear. It is only due to growth via the levels of unbound Tkv receptor that exponential domain growth occurs: this is something that could be used to understand the growth mechanisms more fully and we discuss this in Sect. 6.

It should also be noted that the approximations tend to slightly over predict growth (See Fig. 9b). This result arises since our approximation assumes that the gradient is in a steady state given by $c(x)=\exp (-\beta x)$, when in fact the initial gradient is less steep, being given by Eq. (50). This leads to a slight over prediction in the number of cells that are able to proliferate due to the gradient in Dpp and hence over prediction of the domain size. The errors are less marked in Case II and in Case III.

Finally, we remark that in order to achieve uniform domain growth throughout the disc we have two crucial requirements: the first is that when cell proliferation does occur, it does so at an almost constant rate (so that $\alpha_{a} \approx \alpha_{g}$ ); and the second is that $c_{a}$ and $c_{g}$ must be such that the disc remains close to Case II (so that we have an almost perfect join between the regions).

\section{Discussion}

In this paper we have presented an initial model that can be used to study growth of the Drosophila wing disc due to an activity gradient of Dpp. We have 
(a)

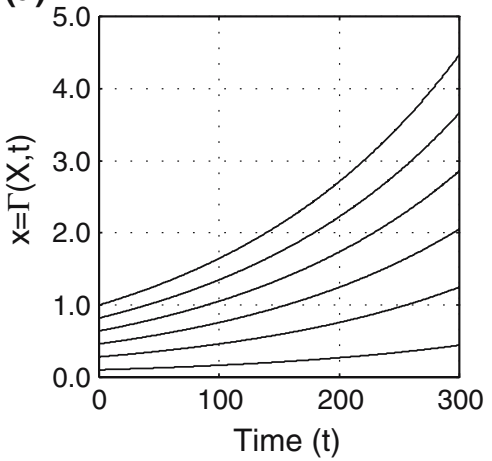

(b)

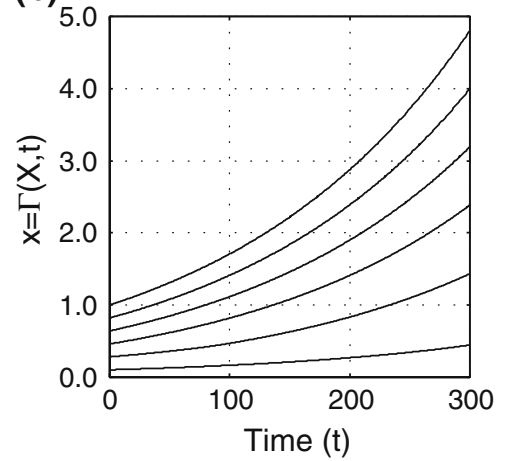

Fig. 10 Analytical approximations for the reduced model as given by Eqs. (52), (53) and (54). a Case II (see Table 2 and Eqs. (55), (56) and (57)) b Case III (see Table 3 and Eqs. (55), (56) and (57)). Parameters are as follows: $D=10.0, \lambda=100, \alpha_{a}=0.005$ and $\alpha_{g}=0.005$. Case II: $c_{s}=0.8$; Case III: $c_{a}=0.7, c_{g}=0.7$

used a system of coupled reaction-diffusion equations with an advection term that describes flow generated in the disc due to cell proliferation. We used a Lagrangian formulation in order to approximate the model numerically and showed that it can generate an exponentially growing wing disc (or in certain cases, a linearly expanding disc). In order to facilitate further analysis, we simplified the model, using a single equation to describe Dpp dynamics. Using this model we were able to find analytical approximations for the rate of growth of the disc and track the movement of cells within the growing domain.

The phenomena observed by Rogulja and co-workers [21] and modelled in the present paper provides one of the most obvious examples in which development is regulated by a threshold response to a morphogen concentration. Although this is an initial model to explain the observed growth phenomena it throws up a number of interesting questions for future work. We describe below a number of avenues that we feel are worthy of future exploration.

Firstly, the extension of the disc boundary is an observable that should be measurable at a number of points over the time scale of disc formation: these data would suggest a qualitative growth law for the disc and could be compared to our model results. Our model predicts either exponential or linear growth rates and it would be interesting to see if this matches experimental observations. Also, estimates of the levels of Dpp and the rates of diffusion and gradient formation would allow for parametrisation of the model and further validation against the observed growth rates.

Secondly, we also note that the system has been perturbed experimentally and that the results of these perturbations may be used to validate the model. Over expression of Dpp leads to increased wing growth, while clonal activation leads to increased proliferation in certain regions of the disc [21]: it would be interesting to see if our model could be adapted to replicate these results and this will be the subject of future work. 
Thirdly, this work considers a very simplified model of a morphogen gradient: without endocytotic trafficking and with very simple forms for terms such as the rate of Dpp diffusion and the internalisation and subsequent decay of Dpp-Tkv. These choices were made in order to build a simple, initial model which captured the essential behaviour of the system: more complex models for Dpp gradient formation and maintenance could of course be designed, but often creating such a complex initial model makes it difficult to elucidate key behaviour and properties, in the manner in which we were able to do so in Sect. 5.

We should also mention that in this work we have concentrated on modelling a Dpp gradient which establishes very quickly (Telemann and co-workers suggest that the rate of gradient formation is about four hours: see, for example, [23]), and where only small levels of flow result from the underlying growth. This allowed us to make certain analytical approximations and also ensured that we did not have problems with our numerical simulations: in mapping our growing domain in $x$ to the fixed domain in $\Gamma$, a fast growth rate results in our effective numerical mesh size quickly increasing and as a result our numerical accuracy decreasing.

Some authors have suggested an alternate view for gradient formation: that in fact the rate of Dpp gradient formation is of the order of about two days; half the time taken for growth of the wing disc [15]. They suggest that initially high levels of unbound Tkv receptor prevent Dpp diffusion (resulting in a steep, short range gradient), but that as Tkv expression is down-regulated a more shallow, long range gradient of Dpp forms. This could also be investigated using similar methods to those employed in Sect. 5 and it would be interesting to see the resulting form of domain growth. We hope to present these results at a later date.

The final question that we feel pertinent to this study is how growth of the domain is regulated. In our model there is no mechanism for termination of growth. It could simply be that cells along the AP segment boundary simply stop expressing Dpp after a certain time, which would eventually dissolve the Dpp gradient, but then we must ask what happens to growth via the brinker pathway. These possibilities remain to be investigated.

Acknowledgements REB would like to thank Lloyds Tercentenary Foundation for a Lloyds Tercentenary Foundation Fellowship, Research Councils UK for an RCUK Academic Fellowship in Mathematical Biology, St Hugh's College, Oxford for a Junior Research Fellowship and Max Planck Institute for Mathematics in the Sciences for a visiting position.

\section{References}

1. Acheson, D.J.: Elementary Fluid Dynamics. Oxford Applied Mathematics and Computing Science Series. Clarendon Press, Oxford (2005)

2. Bollenbach, T., Kruse, K., Pantazis, P., González-Gaitán, M., Jülicher, F.: Robust formation of morphogen gradients. Phys. Rev. Lett. 94(1), 018,103-1-018,103-4 (2005)

3. Briscoe, J., Chen, Y., Jessel, T.M., Struhl, G.: A Hedgehog-insensitive form of Patched provides evidence for direct long-range morphogen activity of Sonic Hedgehog in the neural tube. Mol. Cell 7, 1279-1291 (2001) 
4. Crampin, E.J., Gaffney, E.A., Maini, P.K.: Reaction and diffusion of growing domains: scenarios for robust pattern formation. Bull. Math. Biol. 61, 1093-1120 (1999)

5. Crampin, E.J., Hackborn, W.W., Maini, P.K.: Pattern formation in reation-diffusion models with nonuniform domain growth. Bull. Math. Biol. 64, 747-769 (2002)

6. deCelis, J.F.: Expression and functions of decapentaplegic and thick veins during the differentiation of the veins in the Drosophila wing. Development 124, 1007-1018 (1997)

7. Eldar, A., Rosin, D., Shilo, B.Z., Barkai, N.: Self-enhanced ligand degradation underlies robustness of morphogen gradients. Dev. Cell 5, 635-646 (2003)

8. Ferguson, E.L., Anderson, K.V.: Decapentaplegic acts as a morphogen to organize dorsal-ventral pattern in the Drosophila embryo. Cell 71(3), 451-461 (1992)

9. Funakoshi, Y., Minami, M., Tabata, T.: $m t v$ shapes the activity gradient of the dpp morphogen through regulation of thickveins. Development 128, 67-74 (2001)

10. Houchmandzadeh, B., Wieschaus, E., Leibler, S.: Establishment of developmental precision and proportions in the early Drosophila embryo. Nature 415, 798-802 (2002)

11. Kruse, K., Pantazis, P., Bollenbach, T., Jülicher, F., González-Gaitán, M.: Dpp gradient formation by dynamin-dependent endocytosis: receptor trafficking and the diffusion model. Development 131(19), 4843-4856 (2004)

12. Lander, A.D., Nie, Q., Wan, F.Y.M.: Do morphogen gradients arise by diffusion? Dev. Cell 2, 785-796 (2002)

13. Lander, A.D., Nie, Q., Wan, F.Y.M.: Spatially distributed morphogen production and morphogen gradient formation. Math. Biosci. Eng. 2(2), 239-262 (2005)

14. Lawrence, P.A., Struhl, G.: Morphogen, compartments and pattern: lessons from Drosophila? Cell 85, 951-961 (1996)

15. Lecuit, T., Cohen, S.M.: Dpp receptor levels contribute to shaping the dpp morphogen gradient in the Drosophila wing imaginal disc. Development 125, 4901-4907 (1998)

16. Lighthill, J.: An Informal Introduction to Theoretical Fluid Mechanics. IMA monograph series; 2. Oxford University Press, New York (1996)

17. Mannervik, M., Nibu, Y., Zhang, H., Levine, M.: Transcriptional coregulators in development. Science 284, 606-609 (1999)

18. Martín, F.A., Pérez-Garijo, A., Moreno, E., Morata, G.: The brinker gradient controls wing growth in Drosophila. Development 131, 4921-4930 (2004)

19. Martín-Castellanos, C., Edgar, B.A.: A characterisation of the effects of Dpp signalling on cell growth and proliferation in the Drosophila wing. Development 129, 1003-1013 (2002)

20. Numerical Algorithms Group, http://www.nag.co.uk/numeric/FL/manual/pdf/D03/d03pef.pdf: D03PEF - NAG Fortran library routine document

21. Rogulja, D., Irvine, K.D.: Regulation of cell proliferation by a morphogen gradient. Cell 123, 449-461 (2005)

22. Tabata, T.: Genetics of morphogen gradients. Nat. Rev. Genet. 2, 620-630 (2001)

23. Telemann, A.A., Cohen, S.M.: Dpp gradient formation in the Drosophila wing imaginal disc. Cell 103, 971-980 (2000)

24. Tickle, C.: Morphogen gradients in vertebrate limb development. Cell Dev. Biol. 10, 345-351 (1999)

25. Tsuneizumi, K., Nakayama, T., Kamoshida, Y., Kornberg, T.B., Christian, J.L., Tabata, T.: Daughters against dpp modulates dpp organising activity in Drosophila wing development. Nature 389, 627-631 (1997)

26. Twyman, R.M.: Developmental Biology. INSTANT NOTES. BIOS, Oxford, UK (2001)

27. Wharton, K.A., Ray, R.P., Gelbart, W.M.: An activity gradient of Decapentaplegic is necessary for the specification of dorsal pattern elements in the Drosophila embryo. Development 117, 807-822 (1993)

28. Wolpert, L.: Positional information and the spatial pattern of cellular differentiation. J. Theor. Biol. 25(1), 1-47 (1969)

29. Wolpert, L., Beddington, R., Jessell, T., Lawrence, P., Meyerowitz, E., Smith, J.: Principles of Development, 2nd edn. Oxford University Press, New York (2002) 